menstrual period, this has been well borne. It is to be distinctly understood, however, that the pessary here brought forward is introduced for no other displacement than retroversion. I have but little experience in its use in other displacements, and do not particularly recommend it for them. However, in Fig. 3 is represented a pessary for prolapsus, which embraces the principles of the retroversion pessary modified.
FIG. 2.

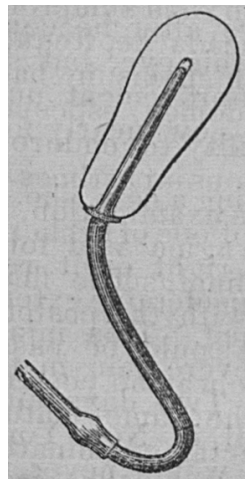

Intra-uterine pessary on a posterior elastio external suspension.
FIG. 3.

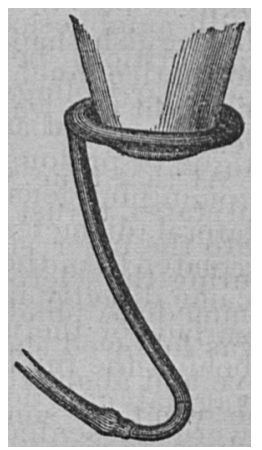

The pelvic band, the India-rubber tube, the jointed hook are the same, but the ring and the single columnar support constitute the modifications. It is not recommended, but suggested, as I have not had a suitable opportunity for its trial.

Both these pessaries can be obtained of Codman \& Shurtleff, 13 and 15 Tremont St., Boston. They are made under the supervision of the writer, and are reliable in workmanship and material.

In conclusion, the writer admits that all pessaries are an evil ; but how can mechanical supports be dispensed with in relieving such a purely mechanical displacement as retroversion of the womb?

December, 1867.

\section{RUPTURE OF THE SCLEROTIC;}

Witn a Case in which the Lens and the whole OF THE IRIS ESCAPED THROUGH THE WOUND, YET tire Patient retained Vision of one tuird, WITH AN APPROPRIATE GLASS.

Read before the Boston Society for Medical Observation, by O. F. WADSWORTH, M.D.

RUPTURE of the sclerotic is placed by writers on ophthalmology among the most fatal in its effects upon vision of the accidents to which the eye is exposed, and this estimate of the severity of the injury is certainly not exaggerated. Though loss of the or- gan may not be the result, the usual position of the wound must necessarily entail grave lesions.

Unless the blow be inflicted by a sharp body, or one moving with considerable force and rapidity, in which case the part injured is that directly impinged upon, the wound is caused by the contre-coup, and is generally at the inner and upper side, and between the insertion of the recti muscles and the cornea, while at the same time it follows more or less exactly the curve of the latter. The anatomical peculiarities of the orbit, and of the sclerotic itselfand its appendages, and in this connection the tendons of the muscles may be considered as such, seem to be amply sufficient to account for the general situation and direction of the wound. That the blow generally comes from the outer side, or from below, is accounted for by the protection afforded in other directions by the projection of the eyebrow and nose. The sclerotic, thickest posteriorly, gradually thins as it comes forward, is thinnest near its equator, and is strengthened anteriorly by the insertion of the recti tendons and their expansion, and the tarso-orbital ligament. This thinning behind the insertion of the tendons is, however, more than made good by the support which it there receives from the tendons and the capsule of Tenon, and the mass of fat and cellular tissue which fills the orbit, and in which the globe lies, must also have a protecting influence on the posterior parts of the scelerotic. The direction of its fibres, which chiefly, and especially in its anterior part, are nearly concentric to the circumference of the cornea, seems to determine the direction of the wound. According to Lawson, the sclerotic may also be ruptured by a blow falling upon the cornea, which, being a segment of a smaller sphere, acts like a wedge, and from its great clasticity may itself escape injury.

This situation of the wound, directly over the attachment of the iris, ciliary processes, and lens, must, almost without possible exception, cause laceration and prolapse of the iris, and, generally, laceration of the ciliary processes, and of the suspensory ligament of the lens, with dislocation of the latter, and if not immediate rupture of its capsule, later changes in its substance. Not infrequently the lens, as well as a portion of the iris, is forced out through the wound, and if, as sometimes happens, its capsule, and the conjunctiva above the wound, be not ruptured, may for a long time present the appearance of a subconjunctival cystic tumor. Always there is 
hæmorrhage into the anterior chamber. Besides these lesions, in a majority of cases there is loss of more or less vitreous, and frequently rupture and separation of the retina and choroid, with hæmorrhage into the posterior part of the eye.

The whole amount of the injury received can, however, not be determined at first; the blood in the anterior chamber prevents the view of the interior of the globe, and it is only after its absorption that the deeper parts can in any case be seen. Though the retina and choroid be uninjured, inflammatory changes in the anterior part of the eye often interfere with the inspection of the fundus even at a later period, and if these structures are also implicated, the probability of ever determining the whole extent of the injury, except after removal of the eye, is naturally much less. Shrinking or suppuration of the globe, and sympathetic ophthalmia, are frequent sequelæ of the injury. By no means in all cases, however, is the final result so fatal ; many cases occur where much useful vision is preserved, and a few even retain sufficient for reading ordinary print.

The treatment of this injury is sufficiently simple. Any portion of iris or vitreous protruding through the lips of the wound and preventing its closure should be removed with scissors, whether underneath the unbroken conjunctiva or not, and in the rare cases where the lens remains beneath the conjunctiva it too should be removed. Rest, preferably in a horizontal position ; the local application of a solution of atropia or of aqueous extract of opium, and thin folds of linen wet with cool water; anodynes internally for relief of pain, are in general all that is required. In some cases slight compression by lint and bandage is of value. If the loss of vitreous, the extent of the laceration, or the evident injury of the internal parts, be so great as to preclude all hope of saving the eye, enucleation should be at once performed, in order to save the patient from the long and painful process of suppuration or shrinking of the globe, and the danger of sympathetic ophthalmia.

While rupture of the sclerotic is general$1 y$ so destructive in its effects, the following case is of interest, as showing an amount of immunity from damage to the retina and choroid, and of reparative power in the eye, apparently incompatible with the injury done to the organ.

Patrick Curran, a laborer, aged 62; was born in Ireland, but has lived in this country for 16 years. His health, except for oc- casional bronchitis the last few winters, has, he states, always been very good, and he appears to be an active man for his age. He came to me last summer at the Dispensary on account of his eyes, and I then fitted him with a glass for his right eye, which he has worn since. Recently I have seen him again and taken the history of his case more fully.

Thirty-five years ago, while working in a quarry in England, a piece of iron struck his left eye, producing immediate loss of sight. Two or three days after he could distinguish shadows with this eye, but did not notice much further improvement until his right eye was injured, now nearly four years ago.

At that time, while feeding a cow, she, as he states, thrust the point of one of herhorns into the inner side of the right orbit, rupturing the sclerotic to a considerable extent. Immediate blindness ensued. This injury was followed shortly by severe pain in the eye and about the brow. Two days after he went to some hospital in New York, where he was told that he would never recover any sight in it, but, was advised to remain there two or three weeks for treatment. This advice he declined, at the suggestion of a companion, who remarked that " the Doctors would not make liars of themselves, and may be they would do something to his eye so that he should not see." For two weeks he kept his bed. The pain in and about the eye continued some six weeks. A wet compress constituted the only treatment. At the end of this time he could distinguish light and dark outlines, and vision afterwards gradually improved. The vision of the lefteye also improved since the injury to the right, and with it he can now count fingers on a dark back ground at a distance of six feet.

In the left eye the conjunctiva is healthy ; the tension is slightly diminished; the cornea is clear; the pupil contracts very little under the influence of light, and dilates imperfectly under atropia ; it is not quite circular, but no synechiæ are apparent, otherwise the iris appears normal. The lens is opaque, showing radiated striation, apparently somewhat shrunken, and slightly movable. On or in the anterior capsule are three or four white, round bodies, about half the diameter of the head of a medium sized pin, separated from the opaque portion of the lens by a thin layer of a transparent medium, and casting on it a well defined shadow with oblique light.

In right eye the conjunctiva is healthy. The cornea shows a very slight haziness 
with oblique light, perhaps only because of the dark back ground. The lens is gone, and every vestige of the iris.

There is a slight cicatrix, about half an inch long, at the upper and inner edge of the sclerotic, about half a line distant from the circumference of the cornea and nearly concentric with it; from near the centre of this cicatrix, another, more marked, runs backward for about a quarter of an inch.

With the ophthalmoscope there are a few floating shreds seen in the vitreous on quick movement of the eye. The whole fundus is distinctly seen. The outline of the optic disc is not quite distinct, nearly the color of the surrounding fundus, and encircled by an incompletely atrophied, irregular zone, a little broader at its outer side, and about one-third the diameter of the disc in width. The retinal vessels are normal. The epithelial layer of the choroid contains very little pigment (he has reddish hair and beard), the pigment of its stroma is also of rather small amount, and it is a little irregularly distributed over the whole fundus, but nowhere in very marked degree. The region of the macula presents nothing special.

With this eye, unaided by a lens, he can count fingers at eighteen inches. With a lens of three and a half inches focal distance, placed one-half inch in front of the cornea, the periphery being covered so as to leave only a central, circular opening, two and a half lines in diameter, and lateral light warded off, he has, however, vision of very nearly one-third, reading No. 50 of Snellen's scale at 16 feet; better than the average result after operation for cataract.

The Transmissibility of Glanders between Man and the Horse. - M. Guyon has recently presented to the French Academy of Sciences a paper on this subject. Some years ago, a captain of artillery at Algiers died of what was ascertained to be a wellcharacterized case of glanders. The blood and fluid of the dead body were inoculated on two mares, a horse, and a mule, four animals unfit for service. The disorder was reproduced on the horse, the mule, and on one of the two mares. It is stated that, long after, a military physician, who had helped to dissect the captain's body, gradually declined in health. His fatal illness was long, and accompanied by peculiar symptoms. It was not until the nature of the disorder had become quite patent that the unfortunate physician recollected his having pricked himself very slightly while dissecting the captain's body.-Lancet.

\section{解ospital 色eports.}

\section{MASSACHUSETTS GENERAL HOSPITAL.}

Surgical Operntions for the week ending February 22d.

Reported by Messrs. Rurus P. Lincols and

Jobiali L. HaIE, Jr.

[Continued from page 104.]

10. Tumor of Leg; Amputation through Knee Joint. Dr. H. J. BigELow.-Male, æt. 18. Six months ago, pain called his attention to a small tumor just below the inner condyle of the femur of the right limb. It rapidly increased, accompanied with pain, until, upon entrance, the circumference of the limb just below the patella was four inches more than at the same point around the left leg. Flexion and extension of the leg increased the pain. The form of the limb, at the points described, resembled the inflammatory knee joint of scrofulous subjects. The most prominent portion of the tumor was situated in the inferior half of the popliteal space, and the subcutaneous veins in that region were much enlarged. His general health was becoming impaired in consequence of the constant pain and anxiety. An incision was made into the most prominent portion of the growth, and a small section excised. Dr. Bigelow stated that "its appearance in connection with the evidence adduced, left no doubt as to its malignancy." He then performed amputation at the knee joint, with subscquent excision of the patella and articular cartilage of the femur; the advantage of this operation being, as was stated, " a complete removal of the leg below the knee without leaving an extensive cartilaginous surface, and without the danger attendant upon a considerable opening of the muscles of the thigh."

11. Painful Stump of Arm; Re-amputation. Dr. H. J. Bigelow.-Entered Aug. 24th, 1867, with a compound comminuted fracture of the forearm, and two transverse fractures of the humerus. The forcarm was amputated just below the neck of the radius. He was discharged Oct. 10th, well. Re-entered Nov. 10th, on account of neuralgia in his stump; the pain was sometimes located anteriorly, and at others posteriorly, but almost constant in the region of the cicatrix. The latter was quite sensitive upon being touched. There was an ulcer of about the size of a dime between the external condyle of the humerus and the olecranon process of the ulna, while the end of the stump was red, swollen and œdematous. The ulcer was healed in a 